\title{
Single-mode operation in the slow-light regime using oscillatory waves in generalized left-handed heterostructures
}

\author{
Kosmas L. Tsakmakidis, ${ }^{\text {a) }}$ Andreas Klaedtke, Durga P. Aryal, \\ Cécile Jamois, and Ortwin Hess \\ Advanced Technology Institute, School of Electronics and Physical Sciences, University of Surrey, Guildford \\ GU2 7XH, United Kingdom
}

(Received 20 June 2006; accepted 25 September 2006; published online 13 November 2006)

\begin{abstract}
The authors present an exact, analytic study of oscillatory modes guided by generalized asymmetric two-dimensional planar heterostructures with negative refractive index in either the core or the cladding. It is shown that, in sharp contrast to normal dielectric configurations, these waveguides always possess a frequency region where the second-order oscillatory mode may exist alone and allow for attaining zero group velocity under weak guidance conditions. In addition the mode has a field distribution that renders it excitable with an end-fire approach, making such structures attractive for applications requiring slow light. Advantages compared to previous methods of slowing or stopping light are discussed. (C) 2006 American Institute of Physics.
\end{abstract}

[DOI: 10.1063/1.2387873]

Structures simultaneously exhibiting negative permittivity and permeability were first analyzed by Veselago. ${ }^{1} \mathrm{He}$ showed that their refractive index is negative and that the vectors $\mathbf{E}, \mathbf{H}$, and $\mathbf{k}$ of a monochromatic plane wave inside them form a left-handed triad. Because of these properties, such materials are often termed as negative-index (NI) or left-handed ( $\mathrm{LH})$ materials, as opposed to ordinary media that are always right handed (RH). Recently, these media have garnered revived interest after the proposal of Pendry et al. that a metamaterial possessing negative magnetic permeability in the microwave region could be constructed with a pair of split-ring resonators. ${ }^{2}$

Important potential applications of LH materials in optics and microwaves, such as the possibility to create superresolving lenses or to improve the performance of biosensoring devices, prompted additional investigations into the properties of specific LH heterostructures. ${ }^{3,4}$ From this research it was shown that LH waveguides support two classes of waves: oscillatory or waveguide modes (OM)/fast waves, which are also found in regular dielectric slab structures, and a rich variety of surface plasmon polariton modes (SPP)/ slow waves. An analytic study of the latter ${ }^{5}$ showed that up to 30 bound SPPs can exist in a generalized LH slab waveguide for all choices of optogeometrical parameters. Although approximately a third of the SPP solutions allow for attaining zero group velocity, their inherent sensitivity to small variations of the media interfaces ${ }^{5}$ may limit their practical use. For this reason, oscillatory modes, which generally have their maxima inside the waveguide core, are more suitable for most conceived applications, including slow light. Nonetheless, they were, until now, described only qualitatively, following ad hoc graphic solution approaches. ${ }^{3,4}$ In this manner, relevant modal properties were either not revealed or not conclusively proven and, importantly, explicit expressions for the cutoff conditions of each mode have not been established yet.

In this letter we report on an extension of waveguide mode theory that considers an asymmetric three-layer slab

${ }^{a)}$ Electronic mail: k.tsakmakidis@surrey.ac.uk heterostructure in which either the guiding region or the cladding region may have a negative refractive index. We derive dimensionless, closed-form expressions for the cutoff(s) of the investigated oscillatory modes that allow for the identification of their existence regions. The modes with negative energy flux that give rise to negative group velocity are identified via an explicit expression for the cycleaveraged total power flow $P_{\text {tot }}$ in the guide. Based on this treatment we rigorously prove that, with judicious choice of parameters, there is a frequency region where the second mode can, at the same time, exist alone and attain zero group velocity. Moreover, we show that the inverted (LH-RH-LH) arrangement supports similar modes as the RH-LH-RH heterostructure but with opposite power flux. It is believed that these are the only slab waveguide structures utilizing homogeneous, isotropic ${ }^{6}$ media that have the potential for singlemode operation in the "slow-light regime."

In the analysis that follows we consider the geometry illustrated in Fig. 1, where all media are assumed to be lossless, ${ }^{7}$ homogeneous, and isotropic. We shall be concerned primarily with $p$-polarized (TM) waves, in which the magnetic field is directed along the $y$ axis. First, we investigate the case where the core is LH with thickness $2 \alpha$, bounded asymmetrically by two RH media that satisfy $n_{2}$ $>n_{3}$. The fields in the guide consist of counterpropagating forward and backward modes trapped within the core by total-internal reflection. For wave guidance to occur the wave components of both types of modes must satisfy the

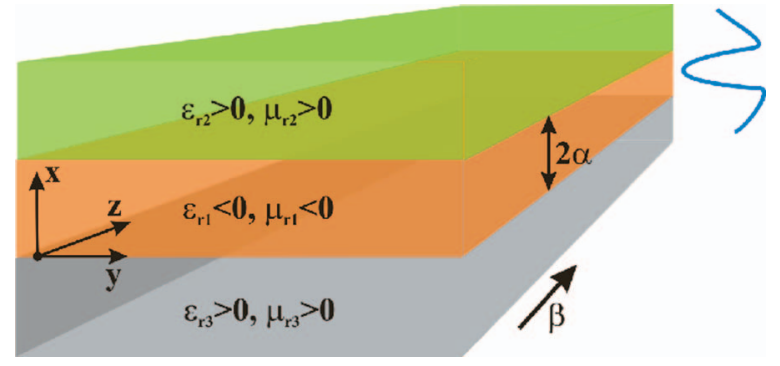

FIG. 1. (Color) Illustration of the asymmetric left-handed planar heterostructure. 

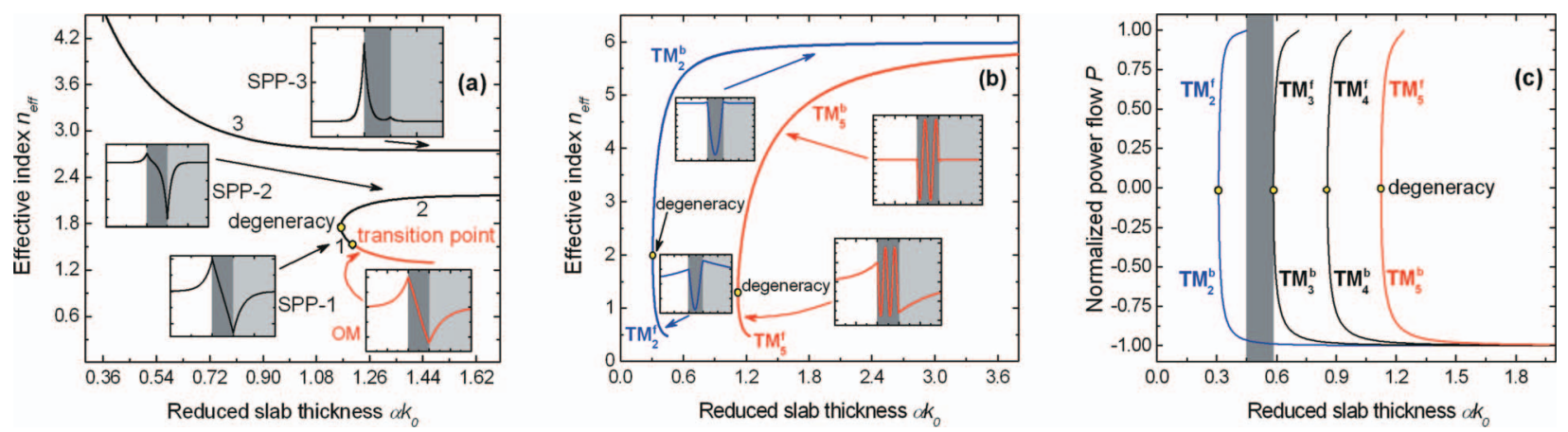

FIG. 2. (Color) (a) Variation of SPP and fundamental oscillatory mode (OM) effective index $n_{\text {eff }}$ with the reduced slab thickness $\alpha k_{0}$ for $\sigma_{\varepsilon}=1.1, \sigma_{\mu}=0.5$, $\rho_{\varepsilon}=1.15, \rho_{\mu}=0.6, \varepsilon_{r 1}=2$, and $\mu_{r 1}=1.2$. (b) Variation of oscillatory mode effective index with reduced slab thickness for $\sigma_{\varepsilon}=\sigma_{\mu}=0.05, \rho_{\varepsilon}=\rho_{\mu}=0.08$, and $\varepsilon_{r 1}=\mu_{r 1}=6$. (c) Variation of oscillatory mode normalized power flow $P$ with reduced slab thickness for the parameters defined in (b).

total-internal reflection condition. This condition can be stated as $\left|n_{1}\right|>n_{\text {eff }}>n_{2}$, where $n_{\text {eff }}$ is the effective index of the mode. We point out here that there is a marked contrast between the present case and that of SPP waves: for the latter case, we require $n_{\text {eff }}>\max \left\{\left|n_{1}\right|, n_{2}\right\}$ as a result of the somewhat different solution ansatz to the wave equation. ${ }^{5}$ Matching the $E_{z}$ and $H_{y}$ fields at the boundaries yields the following dimensionless characteristic equation for oscillatory modes in generalized LH slab waveguides:

$$
\begin{aligned}
\alpha k_{0}= & \frac{1}{2\left|n_{1}\right| \sqrt{1-\rho_{\varepsilon} \rho_{\mu}} b}\left[\arctan \left(\frac{\rho_{\varepsilon} b}{\sqrt{1-b^{2}}}\right)\right. \\
& \left.+\arctan \left(\frac{\sigma_{\varepsilon} b}{\sqrt{t^{2}-b^{2}}}\right)+m \pi\right],
\end{aligned}
$$

where, similar to a recent work, ${ }^{5}$ the following ratios are introduced:

$$
\begin{aligned}
& b\left(n_{\mathrm{eff}}\right)=\frac{U}{V_{2}}=\left[\frac{1-\left(n_{\mathrm{eff}} / n_{1}\right)^{2}}{1-\rho_{\varepsilon} \rho_{\mu}}\right]^{1 / 2}, \\
& t=\frac{V_{3}}{V_{2}}=\left(\frac{1-\sigma_{\varepsilon} \sigma_{\mu}}{1-\rho_{\varepsilon} \rho_{\mu}}\right)^{1 / 2},
\end{aligned}
$$

with $\quad \rho_{\varepsilon}=\varepsilon_{r 2} /\left|\varepsilon_{r 1}\right|, \quad \rho_{\mu}=\mu_{r 2} /\left|\mu_{r 1}\right|, \quad \sigma_{\varepsilon}=\varepsilon_{r 3} /\left|\varepsilon_{r 1}\right|, \quad \sigma_{\mu}$ $=\mu_{r 3} /\left|\mu_{r 1}\right|$, and $k_{0}$ being the free-space wave number. In this work, the two $V$ parameters are defined as $V_{2}\left(\alpha k_{0}\right)$ $=\alpha k_{0}\left(\varepsilon_{r 1} \mu_{r 1}-\varepsilon_{r 2} \mu_{r 2}\right)^{1 / 2}$ and $V_{3}\left(\alpha k_{0}\right)=\alpha k_{0}\left(\varepsilon_{r 1} \mu_{r 1}-\varepsilon_{r 3} \mu_{r 3}\right)^{1 / 2}$, whereas $U=\alpha \kappa=\alpha k_{0}\left(\varepsilon_{r 1} \mu_{r 1}-n_{\text {eff }}^{2}\right)^{1 / 2}$, with $\kappa$ standing for the transverse component of the wave vector in the core. It is noteworthy that similar dispersion expressions, cast in an "inverted" form that is obtained following a zigzag-ray model analysis, describe oscillatory modes in standard asymmetric optical waveguides. ${ }^{8}$ The parameters used in Eqs. (2) and (3) here, though, obey the restrictions $0 \leqslant b \leqslant 1$ and $t$ $>1$. The equalities for $b$ hold at the mode-cutoff points. For each mode $(m=0,1, \ldots)$ and refractive index distribution, the dispersion diagrams, $n_{\text {eff }}$ versus the reduced slab thickness $\alpha k_{0}$, may be directly obtained by noting that $b$ in Eq. (1) is a function of $n_{\text {eff }}$, which in turn increases monotonically from $n_{2}$ to $\left|n_{1}\right|$.

In discussing the cutoff conditions, it is important to recognize the fact that several double mode-degeneracy points can, in principle, appear in the dispersion diagrams since some of the modes in the LH heterostructure will be backward propagating, having antiparallel phase $\left(v_{\mathrm{ph}}\right)$ and group $\left(v_{g}\right)$ velocities. The cycle-averaged total power flow $P_{\text {tot }}$ at Downloaded 30 Mar 2009 to 131.227.178.132. Redistribution subje these (cutoff) points will vanish. It is therefore useful to derive a general closed-form expression for $P_{\text {tot }}$. Integrating the $z$ component of the complex pointing vector over the guide's cross section, ${ }^{5}$ considering Eq. (1), we obtain the following after some algebraic manipulations:

$$
\begin{aligned}
& P_{3}=C \frac{1}{\left|\varepsilon_{r 1}\right|} \frac{\alpha \beta}{\sigma_{\varepsilon} W_{3}} \\
& P_{1}=C \frac{\alpha \beta}{\left|\varepsilon_{r 1}\right|} \frac{W_{3}^{2}+\sigma_{\varepsilon}^{2} U^{2}}{\sigma_{\varepsilon}^{2} U^{2}}\left(\frac{\rho_{\varepsilon} W_{2}}{W_{2}^{2}+\rho_{\varepsilon}^{2} U^{2}}+\frac{\sigma_{\varepsilon} W_{3}}{W_{3}^{2}+\sigma_{\varepsilon}^{2} U^{2}}-2\right) \\
& P_{2}=C \frac{\alpha \beta}{\left|\varepsilon_{r 1}\right| \rho_{\varepsilon} W_{2}} \frac{\rho_{\varepsilon}^{2}}{\sigma_{\varepsilon}^{2}} \frac{W_{3}^{2}+\sigma_{\varepsilon}^{2} U^{2}}{W_{2}^{2}+\rho_{\varepsilon}^{2} U^{2}}
\end{aligned}
$$

where $P_{i}(i=1,2,3)$ is the power confined in the waveguide $i$ layer (Fig. 1), $\beta$ and $W_{j}(j=2,3)$ the mode longitudinal propagation and decay constants, respectively, and $C$ an arbitrary positive constant. It is inferred from Eqs. (4a)-(4c) that the net power flow can become negative in the core layer, but remains positive in the cladding regions. Calculating the total power $P_{\mathrm{tot}}=\sum_{i=1}^{3} P_{i}$, in terms of the dimensionless parameters defined before and the reduced slab thickness, we arrive at

$$
\begin{aligned}
P_{\mathrm{tot}}= & C \frac{W_{3}^{2}+\sigma_{\varepsilon}^{2} U^{2}}{\sigma_{\varepsilon}^{2} U^{2}} \frac{\left[n_{1}^{2}\left(\alpha k_{0}\right)^{2}-U^{2}\right]^{1 / 2}}{\left|\varepsilon_{r 1}\right|}\left(\frac{\rho_{\varepsilon}}{W_{2}} \frac{V_{2}^{2}}{W_{2}^{2}+\rho_{\varepsilon}^{2} U^{2}}\right. \\
& \left.+\frac{\sigma_{\varepsilon}}{W_{3}} \frac{V_{3}^{2}}{W_{3}^{2}+\sigma_{\varepsilon}^{2} U^{2}}-2\right) .
\end{aligned}
$$

To facilitate the discussion of the mode-cutoff relationships, we first note from Eq. (1) that the upper branches $(b$ $\rightarrow 0)$, for all modes other than the fundamental $(m \neq 0)$, show no upper cutoff thickness, since $b \rightarrow 0$ in this case yields $\alpha k_{0} \rightarrow \infty$. The situation is reminiscent of OMs in normal dielectric slab structures and is illustrated in Fig. 2(b). However, for the fundamental mode $(m=0)$ inspection of Eq. (1) reveals a somewhat unusual behavior and a low cutoff point given by

$$
\left.\left(\alpha k_{0}\right)\right|_{\text {low }} ^{m=0}=\frac{1}{2\left|n_{1}\right|}\left(\frac{\rho_{\varepsilon}}{\sqrt{1-\rho_{\varepsilon}^{2} \rho_{\mu}^{2}}}+\frac{\sigma_{\varepsilon}}{\sqrt{1-\sigma_{\varepsilon}^{2} \sigma_{\mu}^{2}}}\right) .
$$

At this transition point, depicted in Fig. 2(a), the SPP-1 mode ${ }^{5}$ transforms continuously to the $m=0$ oscillatory mode, whose field pattern closely resembles that of a surface wave. to AIP license or copyright; see http://apl.aip.org/apl/copyright.jsp 
In Ref. 9, the existence of such cutoff point for the $m=0$ oscillatory mode in asymmetric three-layer LH heterostructures has been associated with the appearance of a complete three-dimensional photonic band gap. Critically, the fundamental mode also exhibits an upper cutoff that can be calculated as

$$
\begin{aligned}
\left.\left(\alpha k_{0}\right)\right|_{\mathrm{upper}} ^{m=0}= & \frac{1}{2\left|n_{1}\right| \sqrt{1-\rho_{\varepsilon} \rho_{\mu}}}\left[\operatorname{atan}\left(\frac{\sigma_{\varepsilon}}{\sqrt{t^{2}-1}}\right)\right. \\
& \left.+\pi\left(m+\frac{1}{2}\right)\right] .
\end{aligned}
$$

This relationship also describes the upper cutoff point of all lower branches in the mode-dispersion diagrams. An exemplary result of such curves for a "slow-light oriented" choice of optogeometrical parameters is shown in Fig. 2(b), with the insets illustrating the corresponding field profiles. For the sake of clarity, the dispersion curve of the fundamental $\mathrm{TM}_{1}$ mode is not shown here, since the upper cutoff point of this mode is $\left.\left(\alpha k_{0}\right)\right|_{\text {upper }} ^{m=0} \cong 0.18763$, i.e., well below the "degeneracy" point of the $\mathrm{TM}_{2}$ branch, which occurs at $\left.\left(\alpha k_{0}\right)\right|_{\mathrm{deg}} ^{m=1}$ $\cong 0.31047$. The proposed nomenclature for recognizing the oscillatory modes consists of a pair of letters to identify the polarization, followed by a subscript to track the number of nodes in the core region and a superscript to designate that the mode is forward $(f)$ or backward (b), i.e., $\mathrm{TM}_{m+1}^{f / b}$ for the $m$ th-order mode.

Figure 2(c) reports the variation of the normalized power flow $P=P_{\text {tot }} /\left(\left|P_{1}\right|+\left|P_{2}\right|+\left|P_{3}\right|\right)$ (Ref. 5) with reduced slab thickness for the previous solutions. One may notice that the two branches of the $\mathrm{TM}_{2}$ mode merge at a critical slab thickness. At this (cutoff) point the total power $P_{\text {tot }}$ of the resulting degenerate mode vanishes and the group velocity reduces to zero. ${ }^{10}$ An exact expression for this cutoff point cannot be given except in the somewhat simplified form of $P_{\text {tot }}=0$. However, for every mode $(m=1,2, \ldots)$ this point can be swiftly calculated following the steps for the derivation of the dispersion diagrams outlined above and requiring $\alpha k_{0}\left(n_{\text {eff }}\right) \rightarrow \min$.

Since the $\mathrm{TM}_{1}$ and $\mathrm{TM}_{2}^{f}$ modes always show an upper cutoff, given by Eq. (7), we conclude that there is a unique and experimentally intriguing region, highlighted by the gray area in Fig. 2(c), where the backward $\mathrm{TM}_{2}^{b}$ mode can, at the same time, exist alone and allow for attaining very small or zero group velocity via adiabatically tapering to the degeneracy point. Under somewhat stronger guidance conditions, i.e., $\rho_{\varepsilon} \rho_{\mu}, \sigma_{\varepsilon} \sigma_{\mu} \rightarrow 0$ for constant cladding indices, it is further possible to altogether suppress the $\mathrm{TM}_{2}^{f}$ branch, thereby increasing the operable width of the highlighted region from approximately 0.134 to 0.274 , for the case shown in Fig. 2(c).

Remarkably, similar modes with reversed power flow are supported by the inverted heterostructure, i.e., one with a RH core (e.g., air) and LH claddings. Indeed, by again assuming a waveguide-mode ansatz for $H_{y}$, i.e., oscillatory field in the core and exponentially decaying field in the cladding regions, and requiring continuity of the tangential field components at the two interfaces, we find that the characteristic Eq. (1) remains unchanged, and so does the expression for the sole magnetic field component $H_{y}$. In a similar vein, it is found that for the new expressions of the cycle-averaged power flow in each layer, one only needs to replace $\left|\varepsilon_{r 1}\right|$ in
Eqs. (4a)-(4c) with $-\left|\varepsilon_{r 1}\right|$. Based on the explicit form of Eq. (1) and since the $V$ parameters defined above are independent of the refractive index sign distribution, it is inferred that all modal properties of the RH-LH-RH arrangement, previously analyzed, are replicated by its "dual" counterpart. It appears at this time that this is the only pair of planar waveguides constructed from isotropic media that can do so. This property also provides increased flexibility in slow-light waveguiding design utilizing LH materials.

In summary, on the basis of an exact, analytic appraisal, it has been shown that an asymmetric planar waveguide utilizing LH media in either the core or the cladding can support single-mode operation in the slow-light regime. The investigated scheme, relying on sufficient decrease of slab thickness, combines the remarkably simple approach for slowing down light suggested in Ref. 11 with the use of efficiently excitable waveguide modes used in Ref. 12, since the profile of the $\mathrm{TM}_{2}$ solution here closely matches that of a single-mode fiber. Moreover, the heterostructures investigated here can be designed to be monomode in the desired frequency range. ${ }^{13}$ The control of the group velocity is achieved by varying the core thickness rather than by varying the temperature or field intensity. ${ }^{12,14}$ The same is true for the light in- and outcoupling, which may be satisfactorily adjusted by adiabatically tapering the size of the waveguide core. We stress that this mechanism for decelerating light does not directly rely on refractive index resonances but merely on the exchange of power between the core and cladding regions, as indicated by Eqs. (4) and (5). Hence, broad band slow light can be obtained provided that the negative material parameters are designed to exist over relatively large bandwidths ${ }^{15}$ at optical frequencies. ${ }^{16}$

This work is partially supported by the Engineering and Physical Sciences Council (EPSRC), UK.

${ }^{1}$ V. G. Veselago, in Proceedings of the First Taormina Research Conference on the Structure of Matter, Pergamon, 1972, edited by E. Burstein and F. De Martini (Pergamon, New York, 1974), p. 5.

${ }^{2}$ J. B. Pendry, A. J. Holden, D. J. Robbins, and W. J. Stewart, IEEE Trans. Microwave Theory Tech. 47, 2075 (1999).

${ }^{3}$ B.-I. Wu, T. M. Grzegorczyk, Y. Zhang, and J. A. Kong, J. Appl. Phys. 93, 9386 (2003).

${ }^{4}$ I. V. Shadrivov, A. A. Sukhorukov, and Y. S. Kivshar, Phys. Rev. E 67, 057602 (2003).

${ }^{5}$ K. L. Tsakmakidis, C. Hermann, A. Klaedtke, C. Jamois, and O. Hess, Phys. Rev. B 73, 085104 (2006).

${ }^{6}$ Th. Koschny, L. Zhang, and C. M. Soukoulis, Phys. Rev. B 71, 121103(R) (2005).

${ }^{7}$ H. Chen, L. Ran, J. Huangfu, X. Zhang, K. Chen, T. M. Grzegorczyk, and J. A. Kong, Phys. Rev. E 70, 057605 (2004).

${ }^{8}$ H. Kogelnic and V. Ramaswamy, Appl. Opt. 13, 1857 (1974).

${ }^{9}$ I. V. Shadrivov, A. A. Sukhorukov, and Y. S. Kivshar, Phys. Rev. Lett. 95, 193903 (2005)

${ }^{10}$ R. Loundon, J. Phys. A 3, 233 (1970).

${ }^{11}$ A. Karalis, E. Lidorikis, M. Ibanescu, J. D. Joannopoulos, and M. Soljačić, Phys. Rev. Lett. 95, 063901 (2005).

${ }^{12}$ Y. A. Vlasov, M. O'Boyle, H. F. Hamann, and S. J. McNab, Nature (London) 483, 65 (2005).

${ }^{13}$ K. L. Tsakmakidis, A. Klaedtke, D. P. Aryal, and O. Hess, Slow and Fast Light 2006 Technical Digest (Optical Society of America, Washington, DC, 2006), Paper No. WA5.

${ }^{14}$ M. D. Lukin and A. Imamoglu, Nature (London) 413, 273 (2001).

${ }^{15}$ H. Chen, L. Ran, J. Huangfu, X. Zhang, K. Chen, T. M. Grzegorczyk, and J. A. Kong, Appl. Phys. Lett. 86, 151909 (2005).

${ }^{16}$ S. Linden, C. Enkich, M. Wegener, J. Zhou, Th. Koschny, and C. M. Soukoulis, Nature (London) 306, 1351 (2004). 